\title{
The usefulness of white light endoscopy, narrow band imaging, and magnification for the optimization of diagnosis in Barrett's esophagus
}

\author{
Boeriu Alina1*, Brusnic Olga1, Onișor Danusia¹, Pascarenco Ofelia1, Boeriu C², Dobru Daniela1 \\ 1 Department of Gastroenterology, University of Medicine and Pharmacy Tirgu Mures, Romania \\ 2 University of Medicine and Pharmacy Tirgu Mures, Tirgu Mures County Emergency Hospital, Tirgu Mures, Romania
}

\begin{abstract}
The diagnosis of dysplasia and early neoplasia in Barrett's esophagus by conventional endoscopy is based on a four- quadrant random biopsies protocol that is prone to sampling errors. Novel endoscopic techniques have been developed to enhance the detection of premalignant and malignant lesions by real time assessment of microvasculare architecture and mucosal structure. Chromoendoscopy with magnification has improved the visualization of lesions, but the dye application impairs a clear evaluation of vascular network. Narrow band imaging endoscopy enhances vascular imaging by using narrow bandwidth lights, with penetration to superficial mucosal structures. Different classification systems of mucosal and vascular patterns have been developed to improve the diagnostic accuracy of non-dysplastic and dysplastic BE, as well as of early esophageal cancer. This article is focused on both the clinical benefits and controversies surrounding conventional and advanced endoscopic methods used for screening and surveillance of patients with Barrett's esophagus. Current evidence shows that the adoption of new technology in routine practice requires a high level of performance as well as the standardization of various classification systems.
\end{abstract}

Keywords: endoscopy, Barrett's esophagus, dysplasia, early cancer

Received: 30 October 2014 / Accepted: 7 December 2014

\section{Introduction}

The early detection and therapy ofesophageal adenocarcinoma represents a substantial public health problem, due to the fact that this neoplasia is now recognized as the fifth leading cause of cancer-related mortality in men all over the world [1]. Its dismal prognosis in advanced stages has lead to an increased interest in early diagnosis, including the detection and surveillance of precursor conditions.

Barrett's esophagus (BE) represents a premalignant condition characterized by the replacement of squamous epithelium that lines the distal esophagus by columnar epithelium containing intestinal metaplasia (IM), as a consequence of gastro-esophageal reflux. Due to the risk of progression through low-grade dysplasia (LGD) to high-grade dysplasia (HGD), early adenocarcinoma and ultimately invasive esophageal cancer, patients with $\mathrm{BE}$ should undergo endoscopic surveillance in order to detect malignant lesions in early stages, while they are amenable to endoscopic therapy. Recent studies have shown that patients with $\mathrm{BE}$ under surveillance program had earlier stage esophageal tumors treated with endoscopic resection or ablation, with better survival ratesthan patients with more advanced disease [2].

Endoscopic diagnosis and surveillance of BE has evolved during the years, from conventional white light endoscopyto new endoscopic techniques that enhance visualization of mucosal and vascular details. Emerging imaging

* Correspondence to: Alina Boeriu

E-mail: aboeriu@gmail.com techniques showed promising results in improving the sensitivity of endoscopic surveillance of premalignant conditions in the gastrointestinal tract [3]. Magnifying endoscopy, in conjunction with chromoendoscopy and narrow band imaging endoscopy, with or without magnification, have proved to be important tools in the assessment of $\mathrm{BE}$, as well as in patients' surveillance and management. Promising results have been reported regarding the diagnosis of dysplasia and adenocarcinoma in BE, but the clinical application of these advanced endoscopic techniques is still under evaluation, due to the diversity of classification systems.

\section{Principles of diagnostic techniques}

The diagnosis of $\mathrm{BE}$ by conventional white light endoscopy (WLE) is based on the location of gastroesophageal (GE) junction and the detection of areas corresponding to columnar epithelium above the junction. These metaplastic areas show salmon or pink color in white light, in contrast to the gray appearance of surrounding squamous epithelium (Figure1).Mucosal changes are not so easy to detect in cases of short-segment $\mathrm{BE}(<3 \mathrm{~cm}$ of metaplastic epithelium lines the esophagus), when the visualization of modified mucosa in white light becomes difficult. Biopsies from the GE junction represent the gold standard for the diagnosis of specialized columnar epithelium that indicates intestinal metaplasia. According to international guidelines $[4,5]$, patients with IM are advised to undergo periodic endoscopic surveillance, for detection of neoplastic changes. The recommended protocol of surveillance using 
WLE consists in 4- quadrant random biopsies taken every 1 to $2 \mathrm{~cm}$ intervals within the $\mathrm{BE}$ and additional targeted biopsies of any suspicious lesions (Seattle protocol). This protocol is difficult to apply in routine practice in long $\mathrm{BE}$ (>3 cm length of metaplastic area), and the diagnosis of dysplasia and early cancer by random biopsies is prone to sampling errors. The poor adherence of patients with long $\mathrm{BE}$ to this protocolrepresents a reality in clinical practice [6]. Some endoscopic methods have been proposed for the improvement of visualization of mucosal changes. Advanced endoscopic techniques have been developed for the improvement in detection of modified areas in $\mathrm{BE}$ and a surveillance protocol based on targeted biopsies from these areas could represent a reliable alternative to the random biopsies approach.

Chromoendoscopy (CHR) with magnification has long been considered a method that improves the diagnostic of BE. This method requires several steps in order to obtain good endoscopic images for the proper evaluation of lesions. A transparent cap is adjusted on the tip of the endoscope. Thus, a distance of $2-3 \mathrm{~mm}$ is maintained between the mucosa under examination and the endoscope, retaining a focused image for detailed inspection. A proper analysis of fine mucosal features requires patient cooperation. This can be achieved by sedation during the procedure, performed by an anesthesiologist with Propofol. A previous examination of esophagus by conventional WLE allows detection of metaplastic areas and of any visible mucosal changes at the GE junction. After that, dye is spread on the mucosa using a spray catheter inserted through the biopsy channel of the endoscope. Methylene blue is a vital stain that is taken up by absorbent tissues and allows the detection of IM at the GE junction. Acetic acid is a weak acid with a $\mathrm{pH}$ of 2.5 used for the enhancement of surface details. After the time interval required for dye fixation, the mucosa is washed with water and the excess dye is removed by suction. Areas of interest are examined with magnifica-

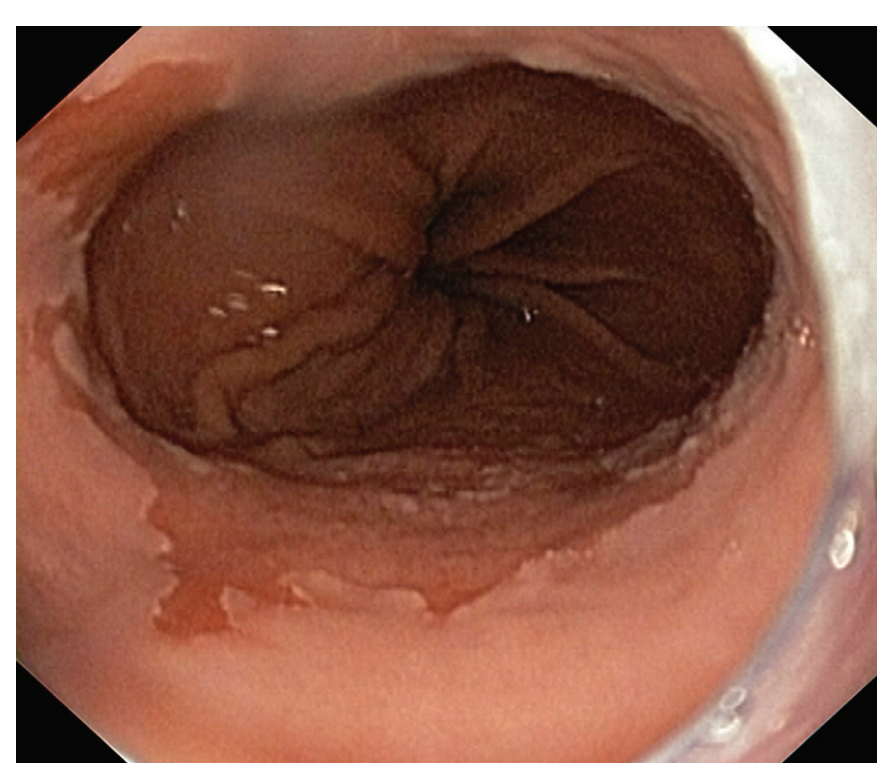

Fig. 1. Long-segment BE in white light endoscopy tion, with a focused evaluation of mucosal and vascular patterns. Targeted biopsies are performed from areas with modified patterns and sent for histological evaluation. As a whole, the method is time consuming and the proper interpretation of different endoscopic images and modified patterns can be difficult.

There are some challenges in the implementation of magnifying CHR in clinical practice. Due to the dye application, sometimes the vascular pattern cannot be properly evaluated. The vascular pattern represents an essential endoscopic feature for the detection of early neoplastic lesions. Another issue is related to the additional time required for the fixation of dye on the mucosa, as well as for washing and removal of excess dye. These potential disadvantages of CHR have been overcome by the application of novel endoscopic techniques, such as narrow band imaging (NBI) endoscopy with magnification.

NBI represents an advanced endoscopic imaging technique, which improves the visualization of mucosal and vascular features by using optical filters. The lights with narrow wavelengths (blue and green) are selected by simply pressing a switch on the high resolution NBI endoscope. These lights penetrate the superficial layers of the mucosa and submucosa and are better absorbed by hemoglobin, leading to the enhancement of vascular details. The red light with deeper penetration in the tissues is taken out. The activation of the magnification function to $150 \mathrm{X}$ improves the visualization of mucosal and vascular patterns. The assessment of neovascularization in dysplastic BE represents an important step in early detection and delineation of malignant lesions.During the examination the endoscopist can freely activate the NBI function and the magnification function of the scope. Images of interest can be frozen to allow a proper assessment of vascular and mucosal patterns. Targeted biopsies can be performed from suspicious areas with distorted patterns, identified by NBI with magnification.

\section{Diagnosis of non-dysplastic and dysplastic Barrett's esophagus and early cancer}

Endoscopic screening of patients for BE detection by WLE has been recommended in various international guidelines, as well as in surveillance strategies [4,5]. Multiple biopsies are advocated to decrease sampling errors, but adherence to this protocol seems to be poor in clinical practice.The selection of high-risk patients for target surveillance could optimize the efficiency of follow-up strategies[7]. The BE length and the grade of dysplasia represent well-known risk factors for the development of esophageal adenocarcinoma. The longer time allocated for careful inspection of $\mathrm{BE}$ seems to be associated with increased detection of HGD and early cancer [8].

In routine clinical practice there arechallenges regarding the accurate diagnosis of dysplasia. The detection of dysplasia can vary between consecutive surveillance endoscopies due to biopsysampling errors, while inter-observer 
agreement among pathologists in diagnosing and grading of dysplasia is poor $[9,10]$. Alternative endoscopic methods have been proposed to improve the accuracy of the diagnosis of dysplasia by performing targeted biopsies from areas with modified patterns.

Magnification CHR has been proven to enhance the detection of specialized IM, and dysplastic lesions on BE. Endo et al. have identified five distinct patterns using methylene blue and magnification: small/round pattern, straight, long oval, tubular and villous pattern. Tubular and villous patterns were specific to IM (Figure 2) [11]. Guelrud et al. have used acetic acid and magnification, socalled "enhanced magnification endoscopy", in order to highlight surface details. They have described four patterns at theGE junction: round, reticular, villous and ridged pattern. Areas showing villous/ridge patterns corresponded with IM (Figure 3) [12].

The distinction between non-dysplastic and dysplastic $\mathrm{BE}$ on magnification CHR is based on the differentiation

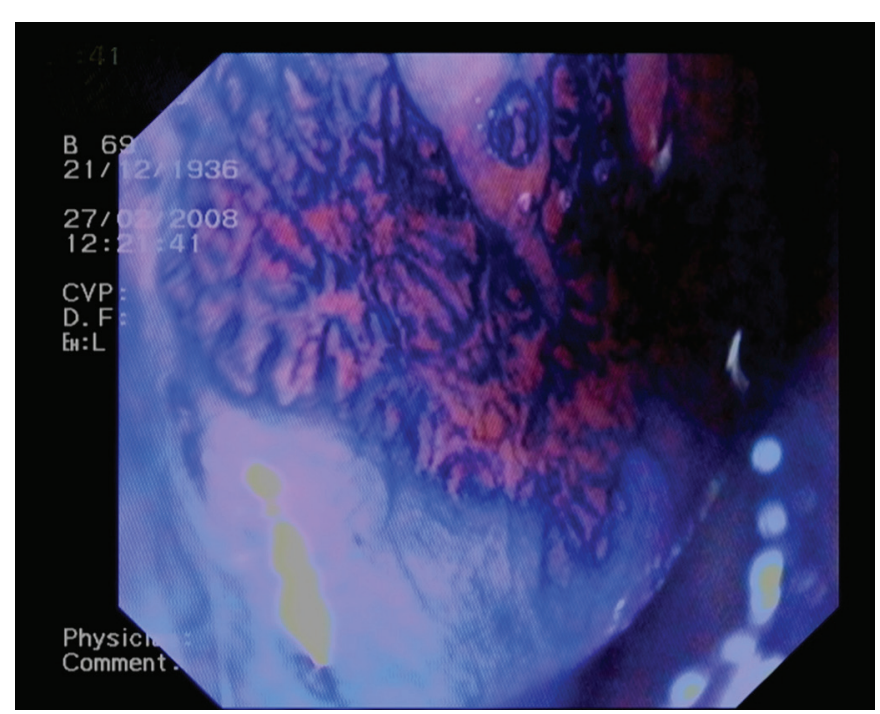

Fig. 2. Chromoendoscopy with methylene blue and magnification: tubular pattern corresponding to intestinal metaplasia (BE)

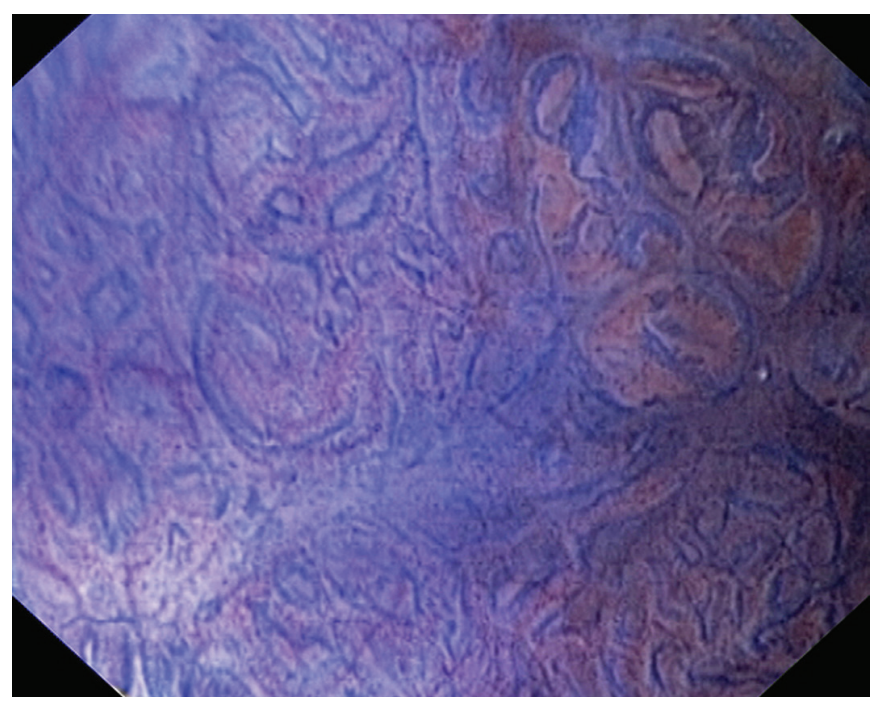

Fig. 4. Chromoendoscopy with methylene blue and magnification: area with irregular/distorted mucosal pattern (dysplastic BE) between regular and irregular/distorted mucosal (Figure 4) and vascular patterns [13]. Because blood vessels may be masked by the use of stains, the main advantage of NBI over CHR is real time assessment of vascularization. With the advancement of NBI endoscopy, clinical studies have been focused on the detection of reliable endoscopic criteria for the diagnosis of dysplasia and early cancer.

Different mucosal patterns have been detected at the GE junction under NBI magnified examination: rounded, circular or oval patterns correspond with columnar mucosa, while flat, villous, and gyrus-shaped patterns are associated with IM (Figure 5) [14]. Irregular/disrupted mucosal patterns, irregular vascular patterns, and abnormal blood vessels have all been described by Kara et al. to be characteristic of high-grade intraepithelial neoplasia HGIN (94\% sensitivity, 76\% specificity, 64\% PPV, 98\% NPV). Regular mucosal and vascular patterns correspond to nondysplastic BE [15]. Singh et al. have described four different patterns on NBI endoscopy with magnification: type

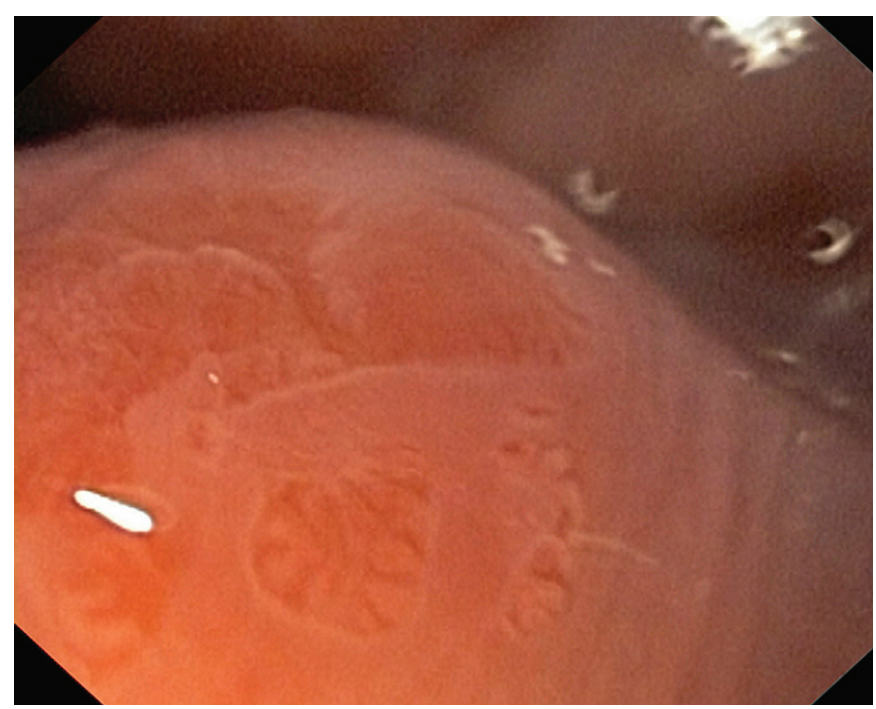

Fig. 3. Chromoendoscopy with acetic acid and magnification: small islands with villous pattern (BE)

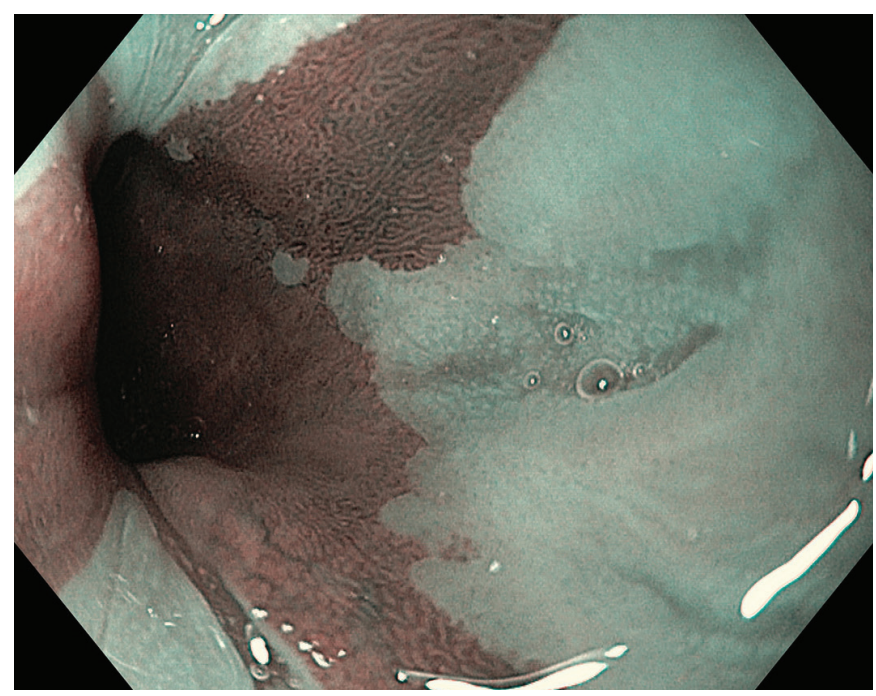

Fig. 5. NBI endoscopy with magnification: regular villous/gyrus pattern with regular vascular pattern (non-dysplastic BE) 
A, consisting of round pits with regular microvasculature, corresponds to columnar mucosa without IM (PPV and NPV were $100 \%$ and $97 \%$ respectively); type B, consisting of villous/ridge pits with regular microvasculature, type $\mathrm{C}$, consisting of absent pits with regular microvasculature, correspond with IM (PPV and NPV were $88 \%$ and $91 \%$ respectively); type $\mathrm{D}$, consisting of distorted pits with irregular microvasculature, is associated with HGD (PPV and NPV were $81 \%$ and $99 \%$ respectively) [16].

NBI endoscopy has proved to be a valuable method for the early detection of neoplastic changes in BE. The irregularity of mucosal and vascular patterns has been described as a distinctive NBI endoscopic feature that is associated with HGD (Figure 6). Wolfsen et al. have demonstrated the superiority of high-resolution NBI endoscopy over standard resolution endoscopy for dysplasia detection in $\mathrm{BE}$ by performing directed biopsies [17]. The diagnostic performance of NBI with magnification for BE and HGD detection has been emphasized by many reports and metaanalysis [18], but the differentiation between LGD and IM remains difficult due to the lack of accurate endoscopic criteria [19].

Despite all the advances, some controversies have been reported regarding the usefulness of NBI in the diagnosis of BE, dysplasia, and early cancer. Thus, an evaluation of the three different classification systems of $\mathrm{BE}$ in a recent meta-analysis has shown that there were some limitations regarding their accuracies for the detection of specialized IM (accuracy ranged between 57\% and 63\%) and dysplasia ( $75 \%$ accuracy), and the inter-observer agreement ranged from fair to moderate [20]. Herrero et al. have studied a simplified classification system for the evaluation of $\mathrm{BE}$ (regular patterns correspond with nondysplastic BE, while irregular patterns correspond with dysplastic $\mathrm{BE}$ ). The results have been disappointing from the point of view of inter-observer agreement and for the diagnosis of HGIN/ early cancer [21].

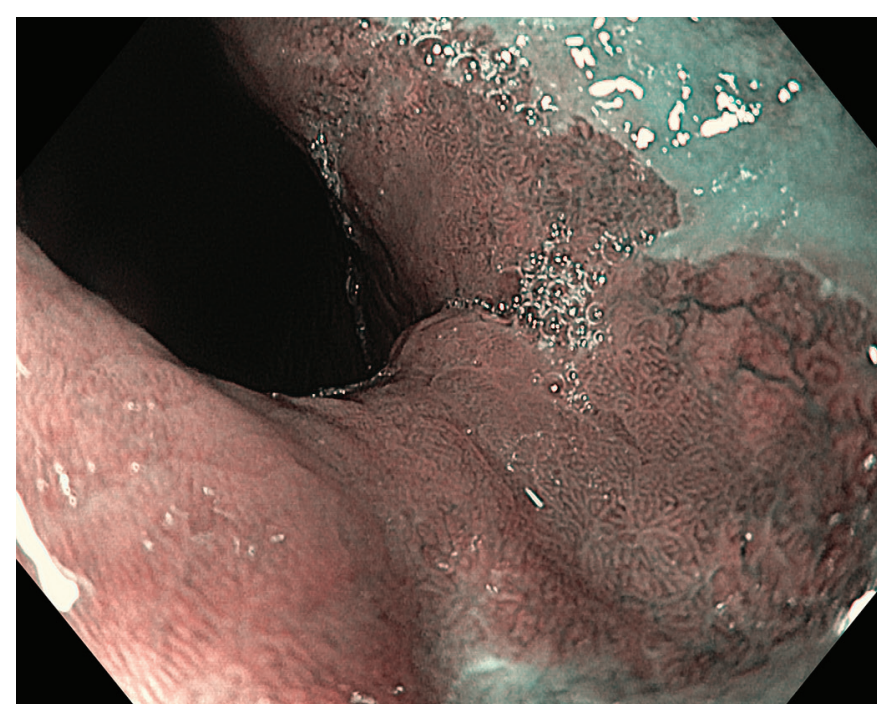

Fig. 6. NBI endoscopy with magnification: irregular mucosal pattern and irregular vascular pattern (high-grade dysplasia)
So far, clinical evidence supports the surveillance protocol based on 4-quadrant biopsies and additional biopsies from suspicious areas, which represents the recommended protocol for the detection of dysplasia and early adenocarcinoma in BE. The newer imaging techniques do not seem to significantly increase the number of patients primary detected with early neoplastic lesions, when compared to high-definition WLE [22]. However, using targeted evaluation of areas with modified patterns,identified by NBI with magnification,by performing directed biopsies, may reduce sampling errors. The delineation of lesions may successfully guide therapeutic endoscopic procedures. The NBI method has proven to be helpful in delineating areas with early neoplasia in BE for subsequent endoscopic mucosal resection. The trimodal imaging evaluation of $\mathrm{BE}$, which combines high-resolution WLE, autofluorescence, and NBI has been discussed to be an alternative to dyespraying techniques for the assessment of early cancer before endoscopic therapy [23]. The endoscopic surveillance of patients after therapeutic procedures could be facilitated by the use of advance imaging technology. In a recent cohort study, the 5 year surveillance after radiofrequency ablation and endoscopic resection for neoplastic BE was successfully performed using high-resolution endoscopy with NBI [24].

\section{Conclusion}

New techniques and improvements in quality may optimize the usefulness of endoscopy for esophageal cancer prevention by facilitating a more efficient screening and surveillance of premalignant lesions. The development of imaging technology enables the detection of specific mucosal and vascular features corresponding to specialized intestinal metaplasia, dysplasia, and early neoplasia in BE. The advantages of the NBI method over CHR consists in the real time assessment of patterns without dye application, which is easier to perform and less time consuming. The interpretation of the images and patterns obtained with these advanced endoscopic methods, or inter-observer agreement, requires a high level of training, expertise, and the utilization of standardized criteria.

The benefit of advanced imaging techniques lies in the real time characterization of lesions, the opportunity to perform focused biopsies, and in the ability to guide therapeutic procedures. NBI endoscopy could represent a more effective procedure in terms of reducing the number of biopsies, improving diagnosis of dysplasia and early cancer, and indirectingendoscopic therapy. At the same time, potential limitations and controversies regarding the clinical application of these techniques have been reported in recent years. Further researchis required to merge the endoscopic criteria into a single, simplified classification system and to establish firm recommendations for clinical application.

Until the validation of these new endoscopic techniques through further clinical trials, the protocol based on con- 
ventional white light endoscopy with multiple biopsies represents the gold standard for diagnosis and surveillance in BE.However, the poor adherence to this protocol, the challenges regarding sampling errors and inter-observer variability among pathologists in dysplasia detection,all support the development of alternative strategies for screening and surveillance.

\section{References}

1. Jemal A, Bray F, Center MM, et al. Global Cancer statistics.CA Cancer J Clin 2011;61:69-90.

2. Grant KS, DeMeester SR, Kreger V, et al. Effect of Barrett's esophagus surveillance on esophageal preservation, tumor stage, and survival with esophageal adenocarcinoma. General Thoracic Cardiovascular Surgery 2013;146:31-37.

3. Ballester V, Cruz-Correa M. Endoscopic surveillance of gastrointestinal premalignant lesions: current knowledge and future directions. Curr Opin Gastroenterol 2014;30:477-483.

4. American Gastroenterological Association medical position statement on the management of Barrett's esophagus. American Journal of Gastroenterology 2011;140:1084-1091.

5. Fitzgerald $R C$, di Pietro $M$, Ragunath $K$, et al. British Society of Gastroenterology guidelines on the diagnosis and management of Barrett's esophagus. Gut 2014;63:7-42.

6. Abrams JA, Kapel RC, Lindberg GM, et al. Adherence to biopsy guidelines for Barrett's esophagus surveillance in the community setting in the United States. Clin Gastroenterol Hepatol 2009;7:736-742.

7. di Pietro M, Fitzgerald RC. Screening and risk stratification for Barrett's esophagus. Gastroenterol Clin N Am 2013;42:155-173.

8. Gupta N, Gaddam S, Wani SB, et al. Longer inspection time is associated with increased detection of high-grade dysplasia and esophageal adenocarcinoma in Barrett's esophagus. Gastrointestinal Endoscopy 2012;76:531-538.

9. Kerkhof M, van Dekken H, Steyerberg EW, et al. Grading of dysplasia in Barrett's oesophagus: substantial interobserver variation between general and gastrointestinal pathologists. Histopathology 2007;50:920-927

10. Curvers WL, ten Kate FJ, Krishnadath KK, et al. Low-grade dysplasia in Barrett's esophagus: overdiagnosed and underestimated. Am J Gastroenterol 2010;105:1523-1530.

11. Endo T, Awakawa T, Takahashi H, et al. Classification of Barrett's epithelium by magnifying endoscopy. Gastrointestinal Endoscopy 2002;55(6):641647.
12. Guelrud M, Herrera I, Essenfeld H, Castro J. Enhanced magnification endoscopy: a new technique to identify specialized intestinal metaplasia in Barrett's esophagus. Gastrointestinal Endoscopy 2001;53(6):559-565.

13. Sharma P, Weston A, Topalowski M, et al. Magnification chromoendoscopy for the detection of intestinal metaplasia and dysplasia in Barrett's esophagus. Gut 2003;52(1):24-27.

14. Kara MA, Peters FP, Rosmolen WD, et al. High-resolution endoscopy plus chromoendoscopy or narrow-band imaging in Barrett's esophagus: a prospective randomized crossover study. Endoscopy 2005;37:929-936.

15. Kara MA, Ennahachi M, Fockens P, et al. Detection and classification of the mucosal and vascular patterns (mucosal morphology) in Barrett's esophagus by using narrow band imaging. Gastrointestinal Endoscopy 2006;64:155-166.

16. Singh R, Anagnostopoulos GK, Yao K, et al. Narrow-band imaging with magnification in Barrett's esophagus: validation of a simplified grading system of mucosal morphology patterns against histology. Endoscopy 2008;40:457-463.

17. Wolfsen HC, Crook JE, Krishna M, et al. Prospective, controlled tandem endoscopy of narrow band imaging for dysplasia detection in Barrett's esophagus. Imaging and Advanced Technology 2008;24-31.

18. Mannath J, Subramanian V, Hawkey CJ, Ragunath K. Narrow band imaging for characterization of high grade dysplasia and specialized intestinal metaplasia in Barrett's esophagus: a meta-analysis. Endoscopy 2010;42:351-359.

19. P, Bansal A, Mathur S, et al. The utility of a novel narrow band imaging endoscopy system in patients with Barrett's esophagus. Gastrointestinal Endoscopy 2006;64:167-175.

20. Silva FB, Dinis-Ribeiro M, Vieth M, et al. Endoscopic assessment and grading of Barrett's esophagus using magnification endoscopy and narrow-band imaging: accuracy and interobserver agreement of different classification systems (with videos). Gastrointestinal Endoscopy 2011;73(1):7-14.

21. Herrero LA, Curvers WL, Bansal A, et al. Zooming in on Barrett oesophagus using narrow-band imaging: an international observer agreement study. Eur J Gastroenterol Hepatol 2009;21:1068-1075.

22. Boerwinkel DF, Swager AF, Curvers WL, Bergman JJGHM. The clinical consequences of advanced imaging techniques in Barrett's esophagus. Gastroenterology 2014;146:622-629.

23. Thomas T, Singh R, Ragunath K. Trimodal imaging-assisted endoscopic mucosal resection of early Barrett's neoplasia. Surg Endosc 2009;23:16091613.

24. Phoa KN, Pouw RE, Van Vilsteren FGl, et al. Remission if Barrett's esophagus with early neoplasia 5 years after radiofrequency ablation with endoscopic resection: a Netherlands cohort study. Gastroenterology 2013;145:96-104. 